\title{
Agent Based Sensor and Data Fusion in Forest Fire Observer
}

\author{
Ljiljana Šerić, Darko Stipaničev and Maja Štula \\ Faculty for Electrical Engineering, Machine Engineering and Naval Architecture \\ Croatia
}

\section{Introduction}

Although natural hazards have been present on earth forever, we still have not developed effective way of dealing with them, and because of that the early detection of natural hazards is still quite important task. Traditional methods based on human observers are mostly used, but they have shown ineffective because of a human observer's subjectivity.

Modern technologies, especially sensors technology provide tools for new techniques of natural hazard early detection. The sensor network technology is one of the most important technologies currently being investigated by scientist all around the world. The sensors, creating a sensor network, today could be deployed in nature on places where it was not possible to put sensors before, particularly because of today's development of wireless communication and miniature autonomous power supplies.

Collecting data from sensors creating a sensor network is one part of the research in this field, and another one is sensor network data utilization. The goal of sensor data interpretation, particularly using data fusion techniques, is to understand the sensor network surrounding and to create the image of the world around the sensors. In this paper this image of the world around the sensors will be call the scenario of the phenomenon.

Combining sensors for collecting data and advanced algorithms for data processing and interpretation, more advanced object called the observer could be designed. Combining several observers an observer network could be established, capable for better understanding what is going on in sensors surroundings. In such a way an advanced early warning system for initial phase natural hazards detection could be designed.

When done by humans, the process of recognition the phenomenon of interest based on fusion of sensory inputs is called perception. Our work was primarily inspired by formal theory of perception introduced in 1989 by Benett, Hoffman and Prakash (Bennett at al., 1989). This theory is used as a framework for formal description of data fusion processes in a observer network.

The observer network is a system consisting of its physical part, various sensors deployed directly in the environment integrated with appropriate communication components, and software part, intelligent mechanisms for sensor network data fusion and interpretation. Data fusion is used as a formal framework for combining data from different physical sensors of the same type, but also from different type of sensors. Data fusion main task is to obtain information of greater quality. The exact definition what 'greater quality' means is primarily application dependent (Wald, 1999). 
Our main phenomenon of interest is forest fire. Forest fires represent a constant threat to ecological systems, infrastructure and human lives. Croatia belongs to countries with enhanced summer forest fire risk, particularly the Dalmatian coast and islands. Great efforts are therefore made to achieve early forest fire detection, which is traditionally based on human surveillance. Since 2003 we have intensively work at University of Split on development of automatic forest fire observer. The forest fire observer is the elementary node of forest observer network currently under development in Croatia, particularly in Croatian National Parks.

\section{The observer theory}

The observer theory is the formal theory of perception (Bennett at al., 1989). The theory is based on thesis that perception is process of inference and that it could be described mathematically using Bayes theorem. The goal of perception is to calculate the probability that specific scenario has occurred if sensory inputs are given; thus the process of perception can be described using Bayesian rule for conditioned probability (Bennett at al., 1996):

$$
P(S \mid I)=\frac{P(I \mid S) P(S)}{P(I)}
$$

where $S$ is the scenario and I is the image, so the interpretation could be that the conditioned probability of occurrence of a scenario S, if the image I is given by sensory input, is equal probability of receiving the image $\mathrm{I}$, if the scenario $S$ happened, multiplied with probability of occurrence of $S$ in the environment divided with probability of receiving the image I. Probability $\mathrm{P}(\mathrm{S})$ depends on the nature of the phenomenon the scenario describes, joined probability $\mathrm{P}(\mathrm{I} \mid \mathrm{S})$ can be induced from sensory mapping functions, i.e. for image camera sensor it is perspective projection together with some limitations of the sensor and $P(I)$ could be available from sensor information type.

According to the theory of perception in real situations this fraction is often uncertain, so more general form of Bayesian theory was used and presented using the formal foundation called the observer (Bennett at al., 1989). The observer is defined as a six-tuple:

$$
\mathrm{O}=(\mathrm{X}, \mathrm{Y}, \mathrm{E}, \mathrm{S}, \pi, \eta)
$$

where $X$ and $Y$ are measurable spaces, $E$ and $S$ are subsets of $X$ and $Y$ respectively, $\Pi$ is a measurable surjective function and $\eta$ conclusion kernel. Space $X$ is a configuration space of the observer and $\mathrm{E}$ is a configuration event of the observer. Space $\mathrm{X}$ is a formal representation of those possible states of affair over which the configuration event $E$ of the observer is defined. $\mathrm{Y}$ is an observation space, or premises space, of the observer. Space $\mathrm{Y}$ is a formal representation of the premises available to the observer for making inferences about occurrences of E. S is the observation event. Only points in $\mathrm{S}$ are premises of observer inferences which conclude that an instance of the configuration event $E$ has occurred. $\Pi$ is a perspective map, the measurable surjective function from $X$ to $Y(\Pi: X \rightarrow Y)$ with $\Pi(E)=S$. $\eta$ is a conclusion kernel of the observer. For each point in the observation event $s \in S, \eta(s, \cdot)$ is a probability measure on E supported on $\left(\Pi^{-1}(s) \cap E\right)$. This means that kernel $\eta$ is a convenient way of assigning a probability measure on $\mathrm{E}$ to every point of $\mathrm{S}$. 


\section{Forest fire observation and the forest fire observer}

\subsection{Traditional approach}

Forest fires are phenomena with devastating consequences. Preferred moment for fighting forest fires is during the initial fire stage. Automatic prediction of enhanced forest fire risk and early detection of forest fire in its initial stage is possible, but it requires a lot of visual, meteorological and historical data, detail information about the monitoring area, and quite complex processing procedures which include a lot of information fusion.

Today the traditional way of information fusion, having as a primarily goal the forest fire detection in early stage, is mostly based on human observers located in monitoring stations on monitoring spots. Human observer collects the data using natural senses, particularly vision, carefully observing his (or her) surroundings, but also reading the data from weather sensors displays and using past experiences and knowledge about particularly vulnerable and dangerous parts of landscape (for example because there is a railway or local road). In specific weather conditions the forest fire risk is enhanced and as a consequence, human observer attention is increased. But finally, the forest fire detection in its initial stage is done by vision. During the fire season a number of human observers, creating the human observers network, are usually responsible for early detection of forest fires in monitoring area. If the fire is spotted, the appropriate fire alarm is raised and the fire-fighters are activated.

\subsection{Observer network approach}

The similar concept was used in our forest fire monitoring observer network designed as a semi-automatic system for raising fire alarms (Stipanicev \& Hrasnik, 2007, Stipanicev et al. 2007a). Forest fire observer network was conceived as a system for real time data monitoring and information fusion having the same goal as the human observers - detection of forest fire in its initial stage. The system is semi - automatic, because the final decision for firefighters activation is done by human operator located in operation center. Now, using the forest fire observer network one operator can take care about larger area in comparison with human observers located on monitoring spots. In forest fire observer network the technical devices called forest fire sensors are located on monitoring spots while processing units and human operator are located in an operation center. The benefit of such system is not only that one operator can control larger area, but also his (her) working conditions in the operation center are much better and lower level of operator concentration is required because the system will warn the operator if any level of hazard is possible.

Forest fire detection in initial stage is based on environmental data collected with the sensor network deployed directly in the environment. Various sensors are responsible for sensing different aspects of reality connected with forest fire phenomenon. The same as in the case of human observers the most important sensor of forest fire observer network is the vision sensor and that is the pan/tilt/zoom controlled CCD camera sensitive in visible spectrum located on appropriate monitoring spot. Additional information are collected using a mini meteorological stations measuring temperature, humidity, wind speed and direction, atmospheric pressure, sun radiation, wetness, lightning discharges etc. An example of our forest fire sensors located in on observatory on mountain Mosor near Split and in Nature Park Vrana lake is shown in Figure 1. 

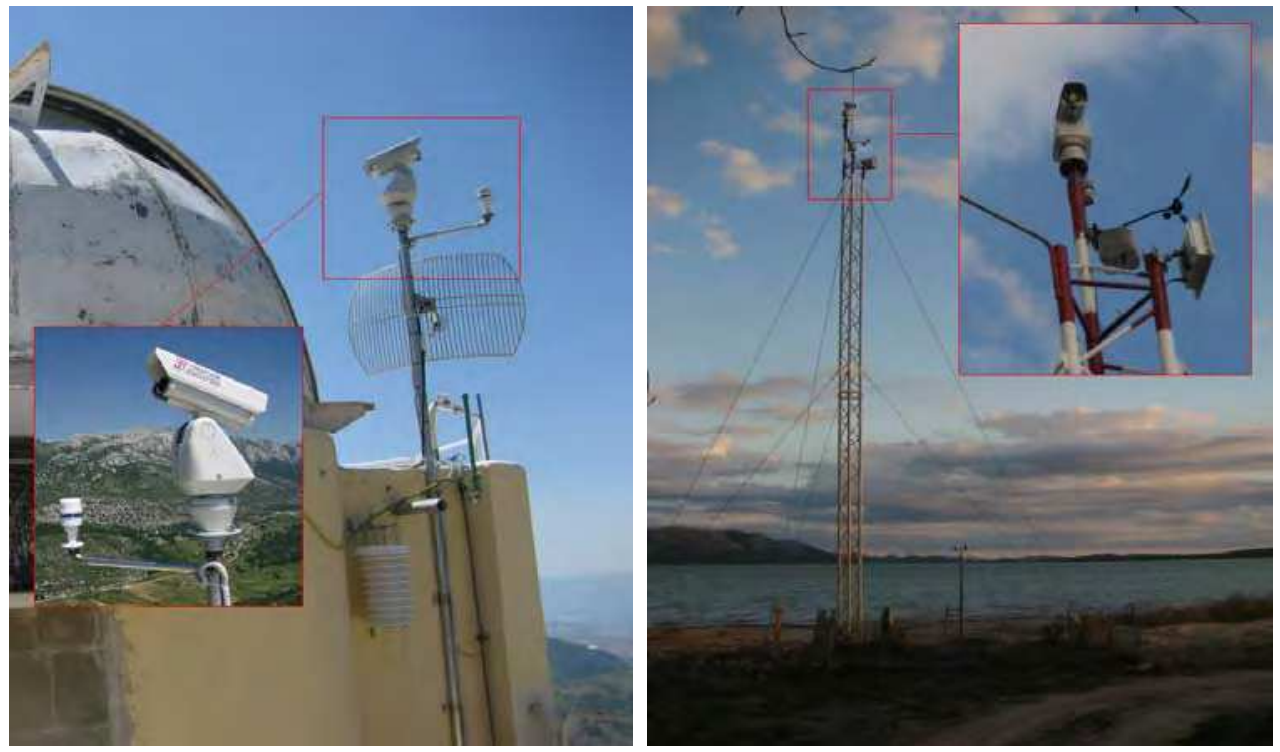

Fig. 1. Forest fire sensors on mountain Mosor near Split and in Nature Park Vrana lake

The main task of the forest fire observer is to collect all these data and to perform algorithms for data validation, data fusion and data interpretation, having as final result information about phenomena taking place in the environment, namely the forest fire in its initial stage. When performed by human observer, these actions are performed using complex processes of perception, so the inspiration to our work was the process of human perception based on the observer theory explained in short in Section 2. Let us here illustrate how observer theory could be applied to the forest fire observer.

Forest fire is a phenomenon of interest which may be recognized from sensory inputs. Simple forest fire observer consists of six entities as stated in equation (2).

The first part is the configuration space $X$. The space $X$ is a set of all possible scenarios that can be recognized in environment. We call this set scene configuration. Some of those scenarios which could happened in our environment observed by forest fire observers are thunder, lightning, twister, fog, forest fire or simple sunny day phenomenon. Only those scenarios holding the occurrence of forest fire are collected in a configuration space E, which is a subset of set $\mathrm{X}$. We referee to $\mathrm{E}$ the name the phenomenon configuration. So set $\mathrm{E}$ holds those scenarios where phenomenon of interest is identified, in our case only the forest fire including all its features, primarily the existence of smoke and flames.

The second part is the observation space Y. Sensors deployed in environment are used to map the input space $X$ to the output space $Y$. We call the space $Y$ the scene observation. The mapping function, or the sensors function is the perspective map п. Depending on the senses available, it can have only elements of orthogonal projection of three dimensional input places to two dimensional image if only a camera or image sensor is available, or it can include some measurements done by other sensors like meteorological sensors. Set $Y$ holds images of scenarios in $X$ with respect of this perspective map. The perspective map has to be injection, but not necessary surjection, so the set $Y$ usually holds less elements then the set $X$. When mapping is done, all elements of $X$ are mapped into elements of $Y$, and 
those elements of $X$ which are also elements of $E$, are mapped into elements of $Y$ which are elements of $S$. The set $S$ is a subset of $Y$ holding those images of scenario where observer can identify phenomenon from $\mathrm{E}$, and we name this set the phenomenon observation. So the conclusion could be: If an image from $S$ is recognized by observer, the observer concludes that the phenomenon has occurred. But the problem is that the perspective map is not surjection, so sometimes happens that a scenario from $E$ (fire) and a scenario from $\neg E$ (not fire) could have the same image inside the set $\mathrm{S}$. So if former scenario (not fire) happens, the observer can falsely conclude that phenomenon had happen. This situation is called the false alarm. The probability of the false alarm is given by the conclusion kernel $\eta$. The kernel $\eta$ gives for each element of $S$ the probability distribution supported on $E$, thus the conclusion kernel gives the final result of our observer - the probability that different scenarios from $\mathrm{E}$ really happen and belong to $S$. The illustration of forest fire observer is shown in Fig. 2.

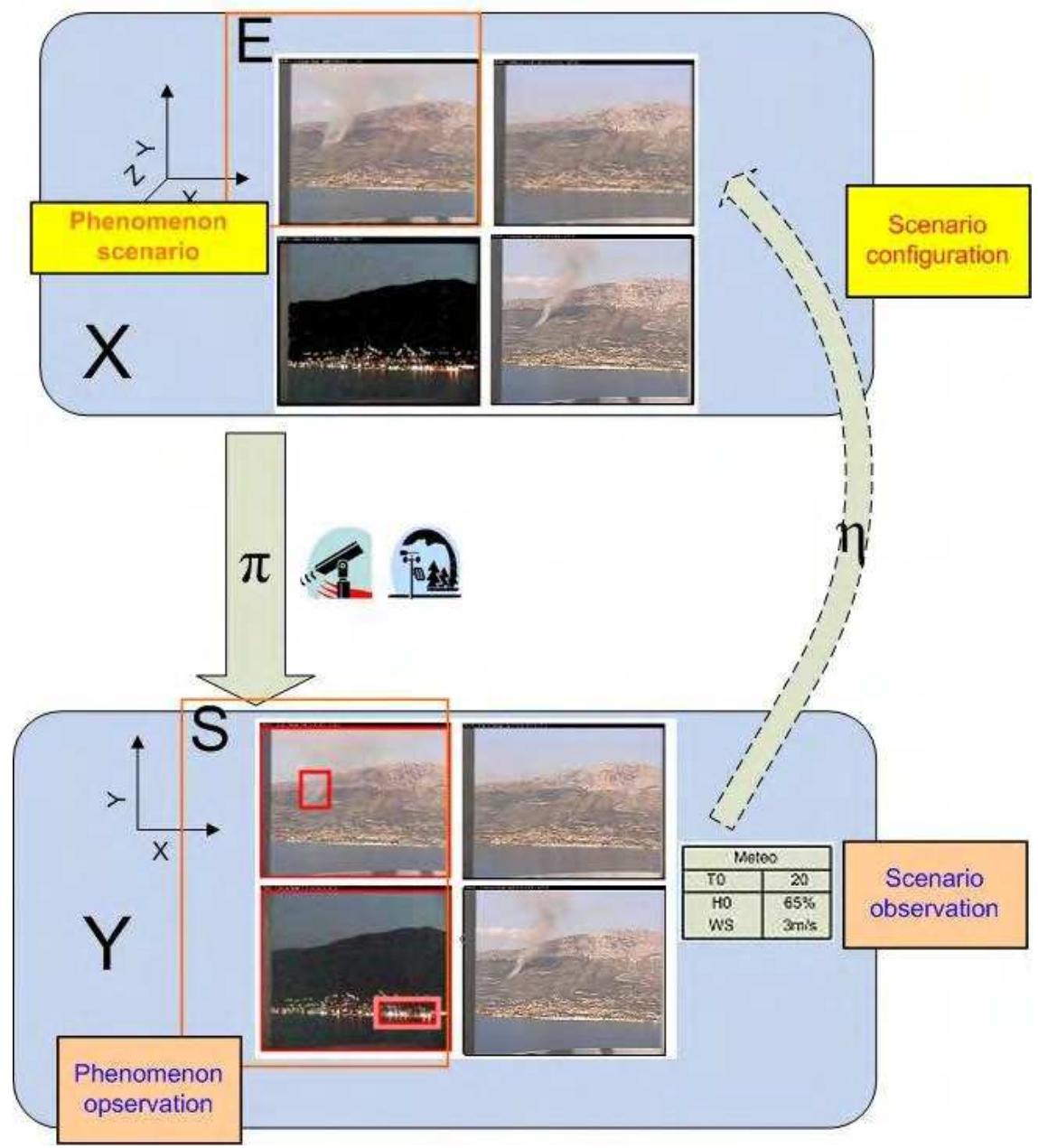

Fig. 2. Illustration of the forest fire observer 
When a phenomenon $x_{1}$ takes place in the environment and it is a forest fire, this event $x_{1}$ is inside the subset $E$ of $X$, so we can write $x_{1} \in E$ (photo in upper left corner of $X$ in Fig.2). In observation space one observation event $\mathrm{y}_{1}$ corresponds to this configuration event (photo in upper left corner of $Y$ in Fig.2). If $y_{1}$ is inside $S\left(y_{1} \in S\right)$, and the observer concludes that this observation event $\mathrm{y}_{1}$ was caused by the configuration event $\mathrm{x}_{1}$ it assigns to pair $\left(\Pi^{-1}\left(\mathrm{y}_{1}\right)\right.$, $\mathrm{x}_{1}$ ) the biggest probability measure. The final observer conclusion is "There is a forest fire" and in decision diagram this situation is treated as a hit or correct detection.

The second case is for phenomenon $\mathrm{x}_{2}$, which is not a forest fire $\left(\mathrm{x}_{2} \notin \mathrm{E}\right.$, photo in upper right corner of $X$ in Fig.2). Let us suppose that the mapped data into observation space are also "clean", without any forest fire signs, so the corresponding observation event ( $\mathrm{y}_{2} \notin \mathrm{S}$, photo in upper right corner of $\mathrm{Y}$ in Fig.2). The final observer conclusion is "There is not a forest fire" and in decision diagram this situation is treated as correct reject.

The third case happens when phenomenon $\mathrm{x}_{3}$ which is outside the subset $\mathrm{E}$ ( $\mathrm{x}_{3} \notin \mathrm{E}$, photo in lower left corner of $X$ in Fig.2) is mapped into an observation event $y_{3}$ which is inside the subset $S\left(y_{3} \in S\right.$, photo in lower left corner of $Y$ in Fig.2). This situation is called the false alarm, because the configuration event is not in $\mathrm{E}$, but the observation event is in $\mathrm{S}$. The conclusion kernel should be defined in such a way that these images are assigned lower probability on $\mathrm{E}$ then in the case of correct detection. The false forest fire alarm could be for example triggered by clouds, clouds shadows or mist.

The forth possible combination is when configuration event $\mathrm{x}_{4}$ is in $\mathrm{E}$ ( $\mathrm{x}_{4} \in \mathrm{E}$, (photo in lower right corner of $X$ in Fig.2) which means that there is a forest fire, but the observation event $y_{4}$ is outside $S\left(y_{4} \in S\right.$, photo in lower right corner of $Y$ in Fig.2). This situation is called the miss case and it is the worst case, taking into question the efficiency of the observer. The phenomenon (forest fire) has happen, but the observer was not able to recognize it. The miss case should be avoided and if the observer is defined well then these situation should never happened.

Four possible decisions of forest fire observer are illustrated in the decision diagram in Fig. 3.

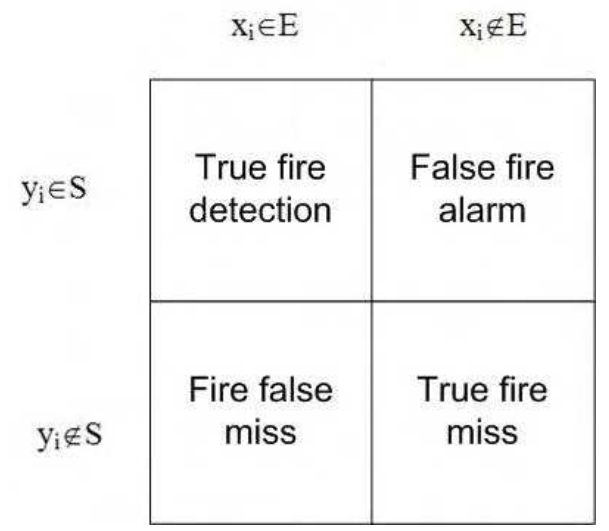

Fig. 3. Decision diagram for the forest fire observer

The quality of the observer could be evaluated according to the number of false alarms and miss cases, and the waiting factor of these two possible decisions could not be the same. In good forest fire observer the set of miss cases has to be the zero set, and the cardinality of the set of false alarms has to be as close as posible to zero. 
The collection of observers form the observer network. The observer network is responsable for phenomena detection in wider area. For example according to our analysis Split and Dalmatia County, which is the costal and island county in Croatia located in central part of Adriatic, having alltogether $14045 \mathrm{~km}^{2}$ and $4572 \mathrm{~km}^{2}$ on land, could be optimaly covered by the forest fire observer network having 56 forest fire sensors (Stipanicev et al. 2007b). Fig. 4 shows their locations and covering. The forest fire sensor on mountain Mosor shown in Fig.1 will be the part of this network, so it is marked in Fig.4. too.

\section{Observer network architecture}

The process of human perception could be divided in two steps: the first one is self perception when observer examines if the readings form his (her) senses are valid (Linsay \& Norman, 1977). If observer in self perception step concludes that there is something wrong with senses, then any other conclusion can not be valid. The second step is identification of scenario based on information from sensors. Having this in mind, we have defined the three layers observer network architecture. Similar architecture was proposed by EU EYES project (EYES, 2005).

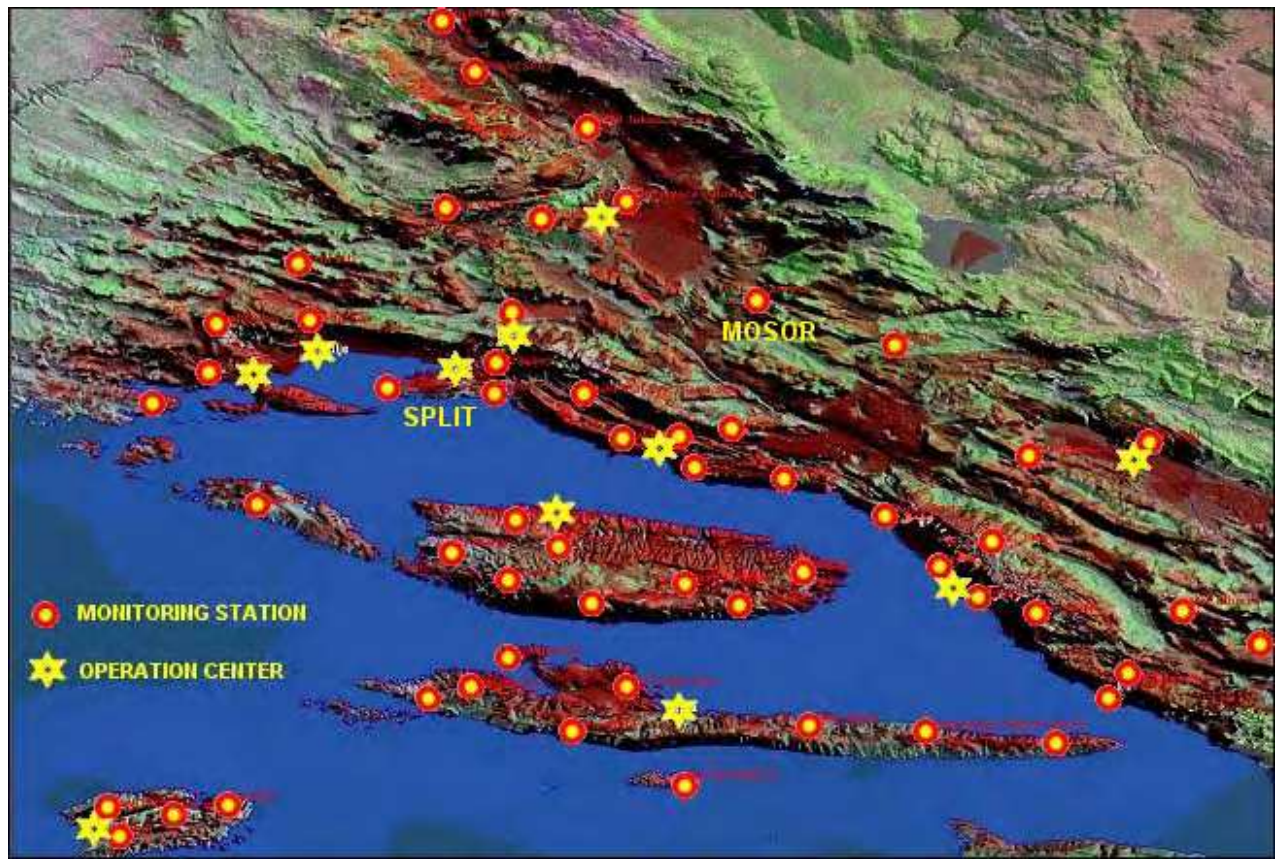

Fig. 4. Forest fire observer network of Split and Dalmatia County

This architecture is designed to be a unified framework for intelligent support of observer network. The framework covers processes of sensor network data collection, validation and implementation in a specific use case. In addition mechanisms for self observation and self reparation are included. The observer network architecture is shown in Fig.5. 


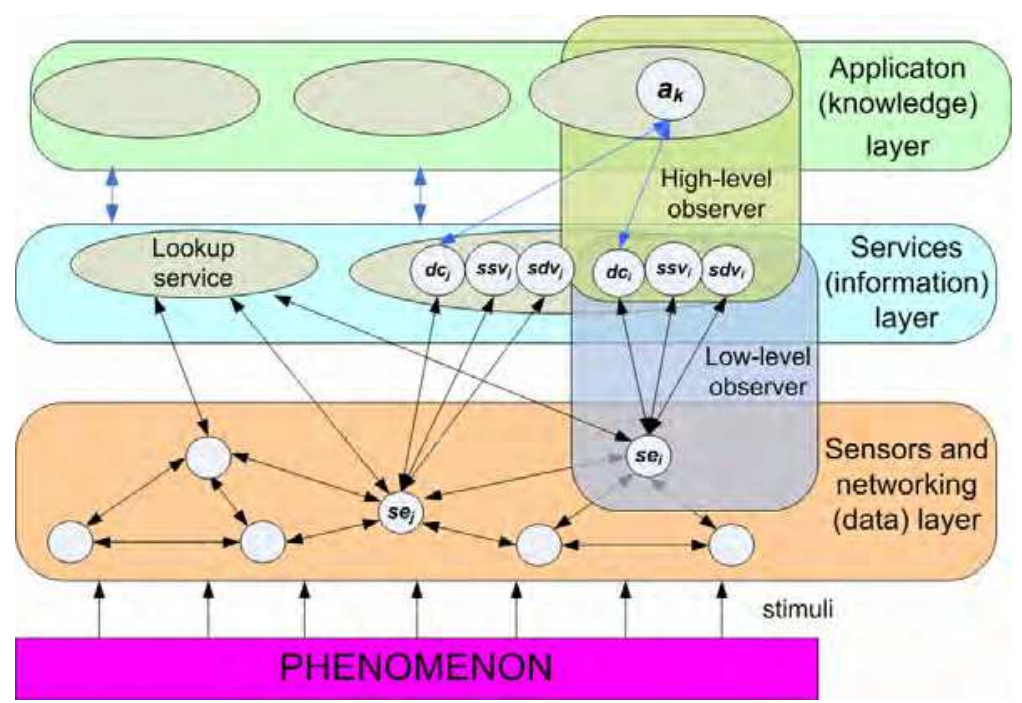

Fig. 5. Illustration of observer network architecture

The information flow is bottom up. Two types of observers has been defined - the low level observer or image fire observer (IFO) and the high level observer or decision fire observer (DFO), both of them composed of elements on two neighboring layers (Stipanicev et al., 2007c).

Low-level observer includes sensors on data layer and processing nodes on information layer. Some aspects of the environment are measured by sensors on data level and transferred as a raw data to nodes on information layer. The sensor $s \boldsymbol{e}_{i}$ is used for mapping the scene configuration space $X$ to first level of scene observation space $Y$ with its own perspective map. On the information layer there are three nodes. The first one $d c_{i}$ is responsible for transformation of raw input data to structured information used as an input of high-level observer. Other processing elements $s s v_{i}$ and $s d v_{i}$ are responsible for observer self perception, performing the validation of data integrity and testing the sensors functionality. Syntactic and semantic validation of data is performed based on the knowledge about the sensor's type and features, as well as their locations. Locations are important because data from other sensors in vicinity of the validated sensor are used during its semantic validation.

High-level observer includes several nodes on information layer and one processing node for each type of observation on the knowledge layer. Let us suppose that only forest fire detection is defined as an observation task. Node $\boldsymbol{a}_{\boldsymbol{k}}$ is responsible for that on knowledge layer, but his decisions are based on information presented to him by various nodes on the information layer. To explain its functionality we will simplify the procedure of forest fire detection and said that it is based on three elements: motion detection, segmentation in RGB space and information is it raining or not. So, forest fire detection node $\boldsymbol{a}_{k}$ on knowledge layer has three corresponding nodes on information layer. Their elements $d c_{i}, d c_{n}$ and $d c_{j}$ are responsible for preparation of appropriate input data. Data presented by nodes $\boldsymbol{d} c_{i}$ and $\boldsymbol{d} \boldsymbol{c}_{n}$ are results of image analysis, and data presented by the third one $d c_{j}$ is a result of moisture sensor row data interpretation. This means that both nodes $d c_{i}$ and $d c_{n}$ are connected with 
the same sensor on the sensor layer and that is the video camera. The third node $d c_{j}$ has a different sensor on the sensor layer and that is the moisture sensor. Both nodes $d c_{i}$ and $d c_{n}$ have the same raw data input (the same digital image), but they transform this input data in different type of output information. Node $d c_{i}$ is a motion detector, so his output data, presented to forest fire decision maker $\boldsymbol{a}_{k}$ are regions on the image where motion has been detected. Node $\boldsymbol{d} c_{i}$ is responsible for RGB segmentation, so his output data presented to forest fire decision maker $\boldsymbol{a}_{k}$ are regions on the image where image red, green and blue components are inside predefined bands. The third node $d c_{j}$ is the most simple one having moisture sensor data as input and binary information 1 (it is raining) or 0 (it is not raining) as an output to $a_{k}$. Now the task of forest fire decision maker is to conclude combining all input data is there a fire on the image or not and if there is a fire where it is located.

On application (knowledge) level it is possible to have various nodes and various applications for example not only forest fire detection, but fog detection, thunderstorm detection, intruder detection, etc. Each of them is connected with various nodes on the services (information) layer. We would like to emphasis that application nodes on knowledge layer make their decisions using data fusion and sensor fusion procedures. Their decisions are based on interpretation of data from various sensors, but also interpretation of data from the same sensor processed in different ways.

\section{Multi agent architecture}

The first part of our work was to define observer network organization, and the second one was its implementation in real life. Before choosing the best environment and architecture for implementation of observer network system we have stated a number of requests, primarily the system has to be modular, suitable to run on distributed environments and controlled through a number of user parameters. Last but not least was that the knowledge base has to be easily accessed and changeable.

Our final choice was the multi agent architecture configurable using database, knowledge base and properties files. Observer network realized in the form of multi-agent system consists of following parts:

- Ontology - defined to ease communication between intelligent agents, and between intelligent agents and external programs. Ontology adds meaning to the database slots.

- Multi agent shell - the core of the system, a shell consisting of agent bodies implementing the observer network functionality. During run time the number of living agents is determined according to the sensor network configuration and system properties written in system files.

- Data base - holds two kinds of tables: administrative tables with the system configuration and data tables with data information and alarms. Each level of observer network architecture has its own result written in its own table.

- Knowledge base - holds additional information, like the common sense rules, about sensors and sensor relative connections. Can be updated during run time, because the system has to be capable to learn.

- $\quad$ Properties file - tells the agents where to find database and external files.

- External programs - virtual sensors can be implemented in the form of formulae or in the form of external programs for more sophisticated calculations and analysis. These external programs can exist independent on the observer network system, but can be used by agent responsible for collecting virtual sensors data. 
Multi agent system, incorporating the three layer architecture is responsible for senor network data collection, verification, and forest fire detection. Intelligent agents are implemented using JADE - Java Agent Development Environment (JADE, 2008). Agents run Rete algorithm in knowledge based reasoning (Singh et al. 2004). The multi agent architecture of the system is shown in Fig. 6.

An agent of type CollectorAgent is responsible for data collection and archiving data into a database. SyntacticObserverAgent is triggered when data is stored into database with purpose of checking syntactic validity. SemanticObserverAgent input are sensor data from the same observer node. This agent first recognizes the same kinds of values on a same observer node, deployed for the redundancy, and notices the difference in them. Small differences are ignored, becouse although the sensors are covering the same location, differences in their exact location, elevation and orientation can cause different values. But larger outliers are rejected.

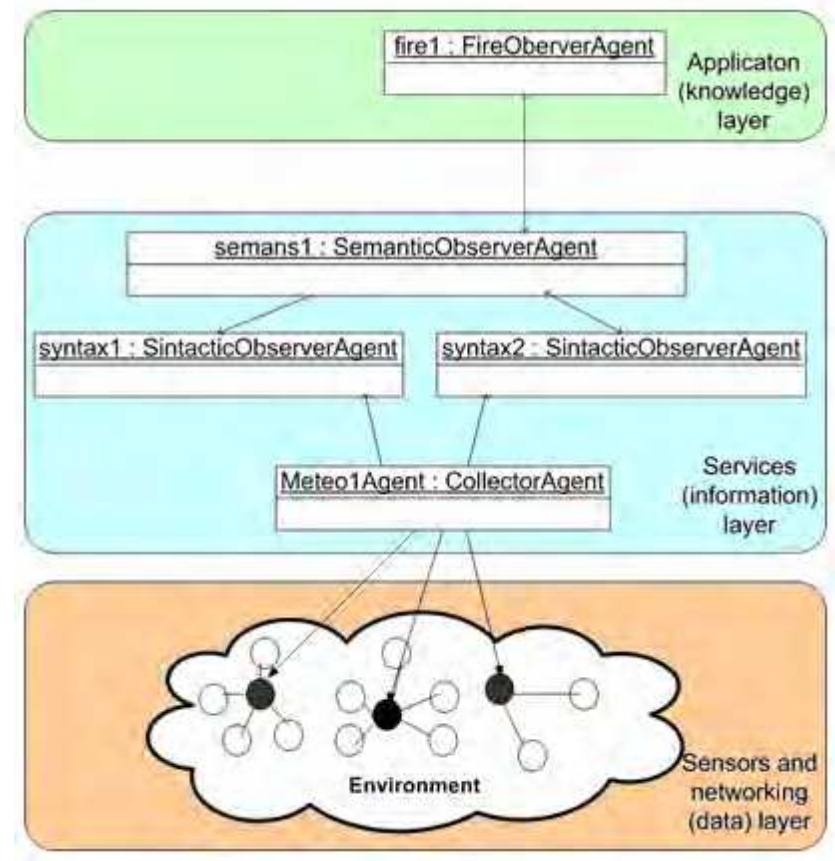

Fig. 6. Multi agent system of the observer network

\subsection{Observer network ontology}

As already stated in previous paragraph, ontology is defined to help the communication between different parts of the system, since those parts are not running in the same process, sometimes not even on the same computer. The ontology of observer networks provides contextual framework to messages that are exchanged between these parts.

Ontology of observer network can be divided in two parts - domain independent and domain dependent part. Domain independent part of ontology holds concepts and formalisms related to sensor and observer network configuration. The concepts of domain 
independent part of the ontology can be linked to layers of three layer observer network architecture, as shown in Fig. 7.

We can consider the topmost concept to be the concept of location which can be compared to the observation station in traditional approach. The concept of location holds all relevant data about the physical location in the environment where the sensor node is situated. Usually one observer node holds one or more Data Server entities with sensing devices attached to it.

Domain dependant part of ontology holds the description of phenomenon taking place in the environment observed. In the case of forest fire observer, this concept holds the description how the forest fire is recognized using sensor values from the sensor network. The most important sensor in this case is the image sensor - video camera sensitive in visible spectra, but meteorological conditions can also be of use in recognition of forest fire and in rejection of false alarm from the image sensor.

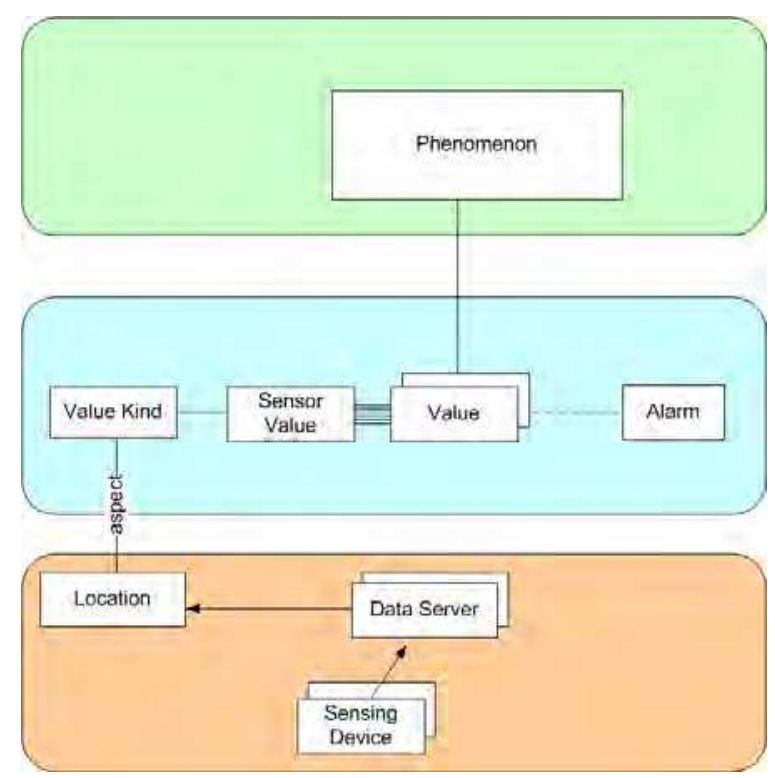

Fig. 7. Concepts of ontology distributed on observer network architecture

\subsection{Multi agent data fusion}

The whole observer network system performs data and information fusion with the goal of constructing the image that is most likely to correspond to the scenario taking place in the environment. Information about the configuration of sensor network and observer network, the knowledge about the phenomenon of interest and data from sensor network are input parts of this fusion and a fusion result is more sophisticated single information - image of scenario.

Following the formalism of observer, configuration space is the space consisting of every phenomenon that can take place in the environment. The configuration event is that phenomenon this observer is responsible for. The sensor network configures the perspective function for mapping different aspects of phenomenon into an observation space consisting 
of data from sensor network and information about sensor network including observer environment. Observation event is the subset of the observation space where phenomenon of interest can be recognized. When an observation of the phenomenon happens, the observer network mechanisms decides which scenario from configuration space is most likely to be the true and that it has happened in the environment.

\section{Conclusion}

Inspirited by the formal theory of perception and technology of sensor network we have introduced the idea of observer network as a reliable framework for data and information fusion. Our ideas have been successfully tested in the case of forest fire observer network. Observer network was implemented using multi-agent technology. A special multi agent shell was designed for this purpose having software system desirable features like modularity and flexibility.

The system was implemented in number of locations in Croatia under the name iForestFire ${ }^{\circledR}$ (iForestFire, 2008), particularly in national and nature parks as a small level observer networks. Two bigger observer networks are now in realization. The first one is in County of Istra where 22 forest fire sensors are already implemented and the second part dealing with multi-agent observer network is now in realization phase. Buzet subsystem is realized and shown in Fig.8. It is conceived of 5 forest fire sensors.

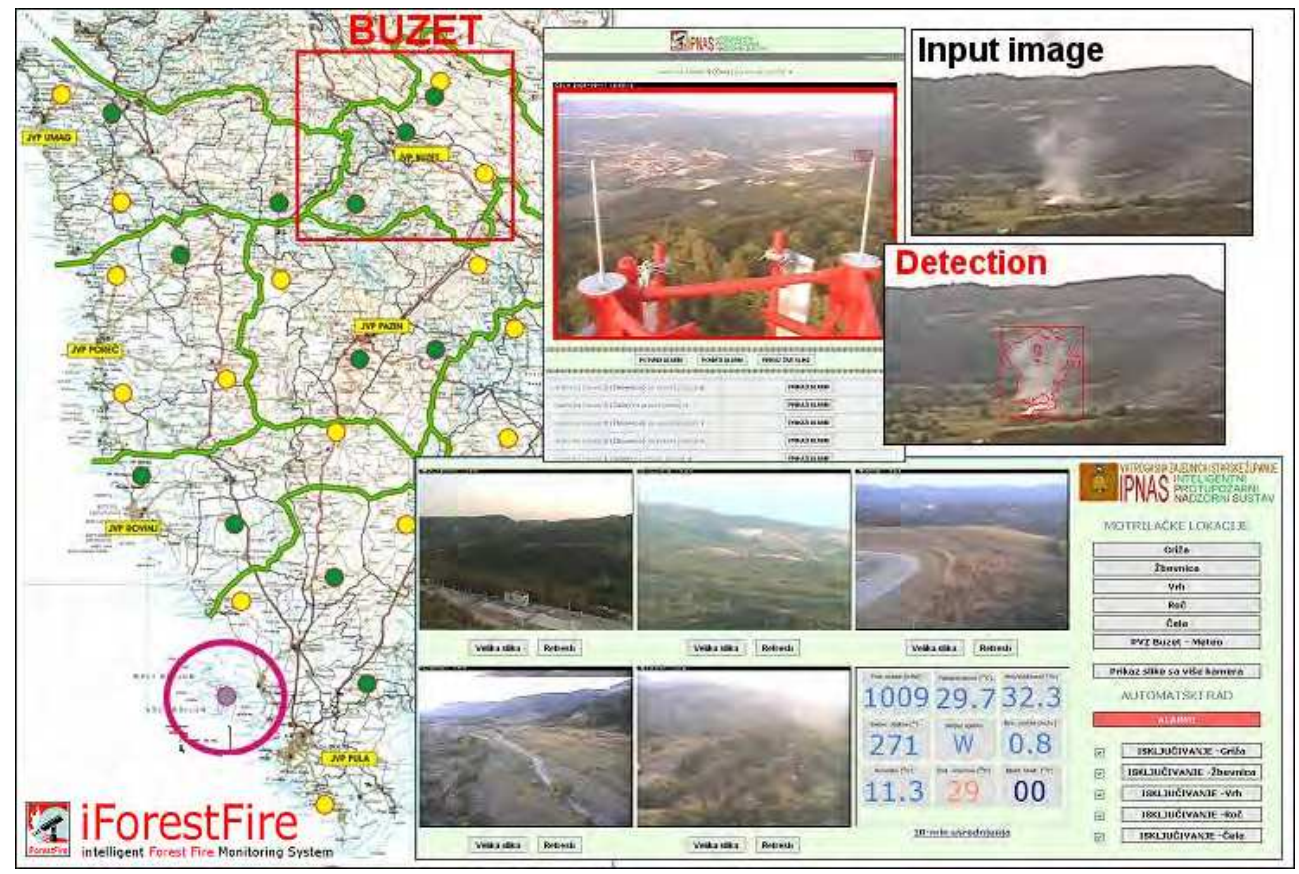

Fig. 8. Forest fire sensors location and various screen of Buzet forest observer network

The Split and Dalmatia County forest fire observer network shown in Fig.4. is also planed for realization. 
Our case study was forest fire detection, so forest fire observer network was designed, but the same concept could be used for recognizing phenomenon of any other kind.

\section{Acknowledgement}

This work was supported by the Ministry of Science, Education and Sport of Republic Croatia under Grant 023-0232005-2003 "AgISEco-Agent-based intelligent environmental monitoring and protection systems", TP-03/0023-09 "System for early forest fire detection based on cameras in visible spectra" and Split and Dalmatia County authorities through a study "Holistic approach to forest fire protection in Split and Dalmatia County“. In this paper only sensor and data fusion principles of forest fire observer network are explain, but for overall system functionality detection algorithms are also important and they were developed and implemented by Damir Krstinić, and Kaja Radić was responsible for experimental systems realization.

\section{References}

Benett, B.M.; Hoffman, D.D. and Prakash, C. (1989). Observer Mechanics - A Formal Theory of Perception", Academic Press Inc., Public Domain

Benett, B.M.; Hoffman, D.D.; Prakash, C. and Richman, S. (1996). Observer theory, Bayes theory, and psychophysics. In D. Knill \& W. Richards (Eds.), Perception as Bayesian inference, Cambridge: Cambridge University Press, pp. 163-212. (UCI MBS Memo 93-12)

Bodrožić,Lj., Stipaničev, D. \& Krstinić, D. (2007) Data Fusion in Observer Networks, MASS 2007, The 4th IEEE International Conference on Mobile Ad-hoc and Sensor Systems, October 8-11, 2007, Pisa, Italy

EYES (2005) Energy Efficient Sensor Network, EU Research Project, 2002 - 2005, http:/ / www.eyes.eu.org

iForestFire (2008) Intelligent Forest Fire Monitoring System, www.iforestfire.com

JADE (2008) Java Agent Development Framework - http://jade.tilab.com

Lindsay, P. \& Norman, D.A. (1977) Human Information Processing: An Introduction to Psychology, Harcourt; 2nd edition

Singh, P.,Minsky, M. \& Eslick, I. (2004). Computing commonsense. BT Technology Journal, 22(4):201-210.

Stipaničev, D. \& Hrastnik, B. (2004) Holistic approach to forest fire protection in Split and Dalmatia County, study for Split-Dalmatia County (220 pages in Cratian)

Stipaničev, D.; Vuko, T., Krstinić, D., Štula, M. \& Bodrožić, Lj. (2006) Forest Fire Protection by Advanced Video Detection System - Croatian Experiences, Third TIEMS Workshop - Improvement of Disaster Management System, Trogir, Sept. 26 - 27, 2006., CD proceeding, 10 pages

Stipaničev, D., Hrastnik, B. \& Vujčić, R., (2007a) Holistic Approach to Forest Fire Protection in Split and Dalmatia County of Croatia, Wildfire 2007 Int. Conference, Sevilla, Spain, May 2007.

Stipaničev, D.; Vuko, T. \& Bodrožić, Lj. (2007b) Location Determination of Automatic Forest Fire Monitoring Stations based on AHP and GIS data, TIEMS (The International Emergency Management Society) 2007 Int. Conference, Trogir, June 2007. CD proceeding, 8 pages 
Stipaničev, D.; Bodrožić, Lj. \& Štula, M. (2007c) Environmental Intelligence based on Advanced Sensory Network, Proceeding of $14^{\text {th }}$ Int. Conf. on Systems, Signals and Image Processing (IWSSIP 2007), Maribor, Slovenia, 27-30.6.2007.

Wald L. (1999) Some terms of reference in data fusion, IEEE Transactions on Geosciences and Remote Sensing, 37 (3), 1190- 1193. 


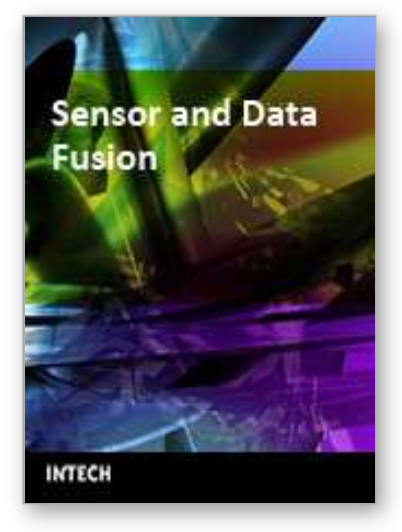

\author{
Sensor and Data Fusion \\ Edited by Nada Milisavljevic
}

ISBN 978-3-902613-52-3

Hard cover, 436 pages

Publisher I-Tech Education and Publishing

Published online 01, February, 2009

Published in print edition February, 2009

Data fusion is a research area that is growing rapidly due to the fact that it provides means for combining pieces of information coming from different sources/sensors, resulting in ameliorated overall system performance (improved decision making, increased detection capabilities, diminished number of false alarms, improved reliability in various situations at hand) with respect to separate sensors/sources. Different data fusion methods have been developed in order to optimize the overall system output in a variety of applications for which data fusion might be useful: security (humanitarian, military), medical diagnosis, environmental monitoring, remote sensing, robotics, etc.

\title{
How to reference
}

In order to correctly reference this scholarly work, feel free to copy and paste the following:

Ljiljana Šerić, Darko Stipaničev and Maja Štula (2009). Agent Based Sensor and Data Fusion in Forest Fire Observer, Sensor and Data Fusion, Nada Milisavljevic (Ed.), ISBN: 978-3-902613-52-3, InTech, Available from:

http://www.intechopen.com/books/sensor_and_data_fusion/agent_based_sensor_and_data_fusion_in_forest_ fire_observer

\section{INTECH}

open science | open minds

\section{InTech Europe}

University Campus STeP Ri

Slavka Krautzeka 83/A

51000 Rijeka, Croatia

Phone: +385 (51) 770447

Fax: +385 (51) 686166

www.intechopen.com

\section{InTech China}

Unit 405, Office Block, Hotel Equatorial Shanghai

No.65, Yan An Road (West), Shanghai, 200040, China

中国上海市延安西路65号上海国际贵都大饭店办公楼 405 单元

Phone: +86-21-62489820

Fax: $+86-21-62489821$ 
(C) 2009 The Author(s). Licensee IntechOpen. This chapter is distributed under the terms of the Creative Commons Attribution-NonCommercialShareAlike-3.0 License, which permits use, distribution and reproduction for non-commercial purposes, provided the original is properly cited and derivative works building on this content are distributed under the same license. 